\title{
Seiberg-Witten invariants and rationality of complex surfaces
}

\author{
Okonek, C ; Teleman, A
}

\begin{abstract}
The purpose of this paper is: 1) to explain the Seiberg-Witten invariants, 2) to show that - on a Kähler surface - the solutions of the monopole equations can be interpreted as algebraic objects, namely effective divisors, 3) to give - as an application - a short selfcontained proof for the fact that rationality of complex surfaces is a $\mathcal{C}^{\infty}$-property.
\end{abstract}

DOI: https://doi.org/10.1007/PL00004300

Posted at the Zurich Open Repository and Archive, University of Zurich ZORA URL: https://doi.org/10.5167/uzh-22248

Journal Article

Accepted Version

Originally published at:

Okonek, C; Teleman, A (1997). Seiberg-Witten invariants and rationality of complex surfaces. Mathematische Zeitschrift, 225(1):139-149.

DOI: https://doi.org/10.1007/PL00004300 


\title{
Seiberg-Witten Invariants and Rationality of Complex Surfaces
}

\author{
Christian Okonek* $\quad$ Andrei Teleman*
}

\section{Introduction}

Recently, Seiberg and Witten introduced new differential invariants for 4manifolds, which are defined by counting solutions of the so called monopole equations, a system of non-linear differential equations of Yang-Mills-Higgs type [18].

The new invariants are expected to be equivalent to the Donaldson polynomial invariants, and they have already found important applications [15].

The purpose of this paper is:

- to explain the Seiberg-Witten invariants

- to show that - on a Kähler surface - the solutions of the monopole equations can be interpreted as algebraic objects, namely effective divisors - to give - as an application - a short selfcontained proof for the fact that rationality of complex surfaces is a $\mathcal{C}^{\infty}$-property.

\section{Spin ${ }^{c}$-structures and the monopole equation}

Definition 1.1 [1], [11] The group $\operatorname{Spin}^{c}(n):=\operatorname{Spin}(n) \times_{\mathbb{Z}_{2}} S^{1}$ is called the complex spinor group.

For the case $n=4$, there is a natural identification

$$
\operatorname{Spin}^{c}(4)=\{(A, B) \in \mathrm{U}(2) \times \mathrm{U}(2) \mid \operatorname{det} A=\operatorname{det} B\} .
$$

*Partially supported by: AGE-Algebraic Geometry in Europe, contract No ERBCHRXCT940557 (BBW 93.0187), and by SNF, nr. 21-36111.92 
The following diagram summarizes some of the basic relations of $\operatorname{Spin}^{c}(4)$ to other groups:

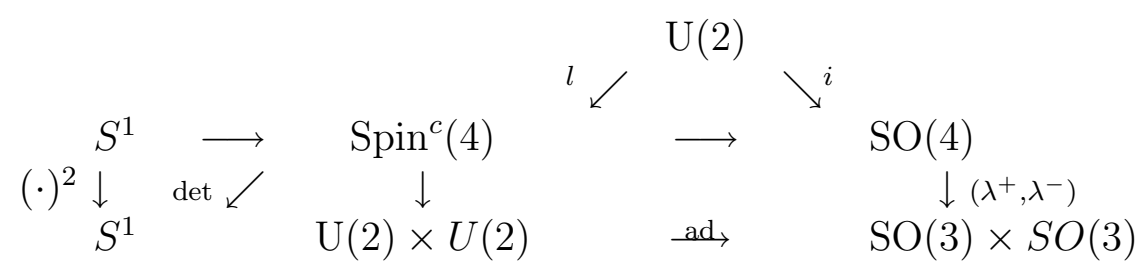

Here $l: \mathrm{U}(2) \longrightarrow \operatorname{Spin}^{c}(4)$ is the canonical lifting of the homomorphism $i \times$ det $: \mathrm{U}(2) \longrightarrow \mathrm{SO}(4) \times S^{1}$ [11], and acts by the formula $\mathrm{U}(2) \ni a \longmapsto\left(\left(\begin{array}{cc}\mathrm{id} & 0 \\ 0 & \operatorname{det} a\end{array}\right), a\right) \in \operatorname{Spin}^{c}(4) . \quad \lambda^{ \pm}: \mathrm{SO}(4) \longrightarrow \mathrm{SO}(3)$ are the maps induced by the two projections of $\mathrm{Spin}(4)=\mathrm{SU}(2)^{+} \times \mathrm{SU}(2)^{-}$onto the factors.

Let $X$ be a closed, oriented simply connected 4 -manifold, $\Lambda^{p}$ the bundle of $p$-forms on $X$, and $A^{p}:=A^{0}\left(X, \Lambda^{p}\right)$ the space of sections in this bundle. Let $g$ be a Riemannian metric on $X$, denote by $P$ the associated principal $\mathrm{SO}(4)$ bundle, and by $P^{ \pm}$the $\mathrm{SO}(3)$-bundles induced via the morphisms $\lambda^{ \pm}$. The real 3-vector bundles $\Lambda_{ \pm}^{2}:=P^{ \pm} \times_{\mathrm{SO}(3)} \mathbb{R}^{3}$ can be identified with the bundles of (anti)self-dual 2-forms, hence there is an orthogonal splitting $\Lambda^{2}=\Lambda_{+}^{2} \oplus \Lambda_{-}^{2}$.

Lemma $1.2[10]$ Given $c \in H^{2}(X, \mathbb{Z})$ with $w_{2}(X) \equiv \bar{c}$ (mod 2) there exists a unique $\operatorname{Spin}^{c}(4)$-bundle $\hat{P}_{c}$ with $P \simeq \hat{P}_{c / S^{1}}$, and $c_{1}\left(\operatorname{det}\left(\hat{P}_{c}\right)\right)=c$.

We denote by $\Sigma_{c}^{ \pm}$the induced $\mathrm{U}(2)$-vector bundles, and we put $\Sigma_{c}:=$ $\Sigma_{c}^{+} \oplus \Sigma_{c}^{-}$.

Lemma 1.3 [1], [11] The choice of a $\operatorname{Spin}^{c}(4)$-lift $\hat{P}_{c}$ of $P$ induces an isomorphism

$$
\gamma_{+}: \Lambda^{1} \otimes \mathbb{C} \longrightarrow \operatorname{Hom}_{\mathbb{C}}\left(\Sigma_{c}^{+}, \Sigma_{c}^{-}\right)
$$

satisfying the identity $\gamma_{+}(u)^{*} \gamma_{+}(v)+\gamma_{+}(v)^{*} \gamma_{+}(u)=2 g(u, v) \operatorname{id}_{\Sigma_{c}^{+}}$for real cotangent vectors $u, v \in \Lambda^{1}$.

We define the homomorphisms $\gamma: \Lambda^{1} \longrightarrow \operatorname{End}_{0}\left(\Sigma_{c}\right), \Gamma: \Lambda^{2} \longrightarrow \operatorname{End}_{0}\left(\Sigma_{c}\right)$ by

$$
\gamma(u):=\left(\begin{array}{cc}
0 & -\gamma_{+}(u)^{*} \\
\gamma_{+}(u) & 0
\end{array}\right)
$$




$$
\Gamma(u \wedge v):=\frac{1}{2}[\gamma(u), \gamma(v)],
$$

and we denote by the same symbols also their $\mathbb{C}$-linear extensions $\Lambda^{1} \otimes \mathbb{C} \longrightarrow \operatorname{End}_{0}\left(\Sigma_{c}\right)$, and $\Lambda^{2} \otimes \mathbb{C} \longrightarrow \operatorname{End}_{0}\left(\Sigma_{c}\right)$. The homomorphism $\gamma$ defines a map $\Lambda^{1} \otimes \Sigma_{c} \longrightarrow \Sigma_{c}$, called the Clifford multiplication. The map $\Gamma$ identifies the bundles $\Lambda_{ \pm}^{2}$ with the bundles of trace free skew-Hermitian endomorphisms of $\Sigma_{c}^{ \pm}$.

Fix a $\operatorname{Spin}^{c}(4)$-bundle $\hat{P}_{c}$ with $P \simeq \hat{P}_{c / S^{1}}$, and let $L_{c}:=\operatorname{det}\left(\hat{P}_{c}\right)$ be the associated $S^{1}$-vector bundle. $L_{c}$ is the unique unitary line bundle with Chern class $c$.

Remark 1.4 [11] The choice of a $S^{1}$-connection $a$ in $L_{c}$ is equivalent to the choice of a $\operatorname{Spin}^{c}(4)$-connection $A$ in $\hat{P}_{c}$ projecting onto the Levi-Civita connection.

Definition 1.5 The composition $\not D_{a}: A^{0}\left(\Sigma_{c}\right) \stackrel{\nabla_{A}}{\longrightarrow} A^{1}\left(\Sigma_{c}\right) \stackrel{\gamma}{\longrightarrow} A^{0}\left(\Sigma_{c}\right)$ is called the Dirac operator associated to the connection $a \in \mathcal{A}\left(L_{c}\right)$.

Notation: Let $\mathcal{A}\left(L_{c}\right)$ be the affine space of $S^{1}$-connections in $L_{c}$. For a connection $a \in \mathcal{A}\left(L_{c}\right)$, we denote by $F_{a} \in A^{2}\left(\operatorname{ad}\left(L_{c}\right)\right)=i A^{2}$ its curvature, and by $F_{a}^{ \pm} \in i A_{ \pm}^{2}$ the components of $F_{a}$ with respect to the orthogonal splitting $A^{2}=A_{+}^{2} \oplus A_{-}^{2}$. Every spinor $\Psi \in A^{0}\left(\Sigma_{c}^{+}\right)$has a conjugate $\bar{\Psi} \in A^{0}\left(\bar{\Sigma}_{c}^{+}\right)$, and we can interpret $\Psi \otimes \bar{\Psi}$ as a Hermitian endomorphism of $\Sigma_{c}^{+}$. Let $(\Psi \otimes \bar{\Psi})_{0} \in A^{0}\left(\operatorname{End}_{0}\left(\Sigma_{c}^{+}\right)\right)$denote the trace-free component of it.

The monopole equations for a pair $(a, \Psi) \in \mathcal{A}\left(L_{c}\right) \times A^{0}\left(\Sigma_{c}^{+}\right)$are the equations [18]:

$$
\begin{cases}\not D_{a} \Psi= & 0 \\ \Gamma\left(F_{a}^{+}\right)= & 2(\Psi \otimes \bar{\Psi})_{0}\end{cases}
$$

Proposition 1.6 (The Weitzenböck formula [11]). Let $s$ be the scalar curvature of $(X, g)$. Fix a $\operatorname{Spin}^{c}(4)$-structure on $X$, and choose a $S^{1}$-connection $a \in \mathcal{A}\left(L_{c}\right)$. Then the following identity holds on $A^{0}\left(\Sigma_{c}\right)$ :

$$
\not D_{a}^{2}=\nabla_{A}^{*} \nabla_{A}+\frac{1}{2} \Gamma\left(F_{a}\right)+\frac{s}{4} \operatorname{id}_{\Sigma_{c}} .
$$


Corollary 1.7 Let $\Psi \in A^{0}\left(\Sigma_{c}^{+}\right)$. Then

$\left.\left\|\not D_{a} \Psi\right\|^{2}+\frac{1}{2} \| \frac{1}{2} \Gamma\left(F_{a}^{+}\right)-(\Psi \bar{\Psi})_{0}\right)\left\|^{2}=\right\| \nabla_{A} \Psi\left\|^{2}+\frac{1}{8}\right\| F_{a}^{+}\left\|^{2}+\frac{1}{4}\right\| \Psi \|^{4}+\frac{1}{4} \int_{X} s|\Psi|^{2}$.

Proof: By the Weitzenböck formula we have

$$
\left(\not D_{a}^{2} \Psi, \Psi\right)=\left(\nabla_{A}^{*} \nabla_{A} \Psi, \Psi\right)+\frac{1}{2}\left(\Gamma\left(F_{a}^{+}\right)(\Psi), \Psi\right)+\frac{s}{4}(\Psi, \Psi),
$$

since $\Gamma\left(F_{a}^{-}\right)$vanishes on $\Sigma_{c}^{+}$; integration over $X$ yields:

$$
\begin{aligned}
\left\|\not D_{a} \Psi\right\|^{2}+ & \left.\frac{1}{2} \| \frac{1}{2} \Gamma\left(F_{a}^{+}\right)-(\Psi \bar{\Psi})_{0}\right) \|^{2}=\int_{X}\left(\not D_{a}^{2} \Psi, \Psi\right)+\frac{1}{2} \int_{X}\left|\frac{1}{2} \Gamma\left(F_{a}^{+}\right)-(\Psi \bar{\Psi})_{0}\right|^{2}= \\
= & \left\|\nabla_{A} \Psi\right\|^{2}+\frac{1}{2} \int_{X}\left(\Gamma\left(F_{a}^{+}\right),(\Psi \bar{\Psi})_{0}\right)+\frac{1}{4} \int \lim i t s_{X} s|\Psi|^{2}+ \\
& +\frac{1}{2} \int_{X} \frac{1}{4}\left|\Gamma\left(F_{a}^{+}\right)\right|^{2}-\frac{1}{2} \int_{X}\left(\Gamma\left(F_{a}^{+}\right),(\Psi \bar{\Psi})_{0}\right)+\frac{1}{4}\|\Psi\|^{4} .
\end{aligned}
$$

Remark 1.8 [18] If $s \geq 0$ on $X$, then the only solutions $(a, \Psi)$ of $(S W)$ are pairs $(a, 0)$ with $F_{a}^{+}=0$.

\section{Seiberg-Witten Invariants}

The gauge group $\mathcal{G}:=\mathcal{C}^{\infty}\left(X, S^{1}\right)$ in the Seiberg-Witten theory is abelian and acts on $\mathcal{A}\left(L_{c}\right) \times A^{0}\left(\Sigma_{c}^{+}\right)$by $(a, \Psi) \cdot f:=\left(a+f^{-1} d f, f^{-1} \Psi\right)$, letting invariant the set of solutions of the equations $(S W)$. We denote by $\mathcal{W}_{X}^{g}(c)$ the moduli space of solutions of the Seiberg-Witten equations, modulo gauge equivalence. A standard technique provides a natural structure of finite dimensional real analytic space in $\mathcal{W}_{X}^{g}(c)$ [6], [5], [16]. The expected dimension of this moduli space is

$$
w_{c}=\frac{1}{4}\left(c^{2}-2 e(X)-3 \sigma(X)\right),
$$

where $e(X)$ and $\sigma(X)$ stand for the Euler characteristic and the signature of the oriented manifold $X$. A solution $(a, \Psi)$ is reducible (has nontrivial stabilizer) if and only if $\Psi=0$, and then the connection a must be antiselfdual. We say that the metric $g$ is $c$-good if the $g$-harmonic representative of the de Rham cohomology class $c_{\mathrm{DR}}$ is not anti-selfdual. If $g$ is $c$-good, then $\mathcal{W}_{X}^{g}(c)$ consists only of irreducible orbits. 
Using the same technique as in Yang-Mills theory ([6], [5]), one defines a gauge invariant perturbation of the Seiberg-Witten equations in order to get smooth moduli spaces of the expected dimension. For a selfdual form $\mu \in A_{+}^{2}$ we denote by $\mathcal{W}_{X}^{g, \mu}(c)$ the moduli space of solutions of the perturbed Seiberg-Witten equations

$$
\begin{cases}\not D_{a} \Psi & =0 \\ \Gamma\left(F_{a}^{+}+i \mu\right) & =2(\Psi \otimes \bar{\Psi})_{0}\end{cases}
$$

We refer to [15] for the following

\section{Lemma 2.1}

1. For every $\mu \in A_{+}^{2}$, the moduli space $\mathcal{W}_{X}^{g, \mu}(c)$ is compact.

2. There is a dense, second category set of perturbations $\mu \in A_{+}^{2}$, for which the irreducible part $\mathcal{W}_{X}^{g, \mu}(c)^{*}$ of $\mathcal{W}_{X}^{g, \mu}(c)$ is smooth and has the expected dimension.

3. If $g$ is c-good, and $\mu$ is small enough in the $L^{2}$ topology, then $\mathcal{W}_{X}^{g, \mu}(c)$ consists only of irreducible orbits, i.e. $\mathcal{W}_{X}^{g, \mu}(c)=\mathcal{W}_{X}^{g, \mu}(c)^{*}$.

4. Let $g_{0}$ and $g_{1}$ be c-good metrics which can be connected by a smooth path of c-good metrics, and let $\varepsilon_{i}>0$ be small enough such that $\mathcal{W}_{X}^{g_{i}, \mu_{i}}(c)=$ $\mathcal{W}_{X}^{g_{i}, \mu_{i}}(c)^{*}$ for all perturbations $\mu_{i}$ with $\left\|\mu_{i}\right\|<\varepsilon_{i}$. Then any two moduli spaces $\mathcal{W}_{X}^{g_{i}, \mu_{i}}(c), \quad i=0,1$, with $\left\|\mu_{i}\right\|<\varepsilon_{i}$, which are smooth and have the expected dimension, are cobordant.

The first assertion is a simple consequence of the Weitzenböck formula and of the Maximum Principle. The other three assertions follow as in Donaldson theory by the Sard theorem for smooth Fredholm maps, and by transversality arguments [5]. Note that in 4. we mean cobordism between non-oriented compact smooth manifolds. A more delicate analysis of the monopole equations [18] shows that, in fact, the moduli spaces $\mathcal{W}_{X}^{g, \mu}(c)^{*}$ come with natural orientations, as soon as they are smooth and have the expected dimension, and that the conclusion in 4 . holds for the oriented moduli spaces.

The Seiberg-Witten theory provides strong differentiable invariants using only moduli spaces of dimension 0 . Let $c$ be an integral lift of $w_{2}(X)$, with $w_{c}=0$, i.e.

$$
c^{2}=2 e(X)+3 \sigma(X) .
$$


Such a lift is called an almost canonical class, since the condition $w_{c}=0$ is equivalent to the existence of an almost complex structure on $X$ with first Chern class $c$ [10], [16].

Now fix an almost canonical class $c$, choose a $c$-good metric $g$, and a small, sufficiently general perturbation $\mu$. Then $\mathcal{W}_{X}^{g, \mu}(c)=\mathcal{W}_{X}^{g, \mu}(c)^{*}$ is compact, smooth of the expected dimension 0, and its bordism class is independent of $\mu$. Let $n_{c}^{g}:=\left|\mathcal{W}_{X}^{g, \mu}(c)\right| \bmod 2$ be the number of points modulo 2 of this moduli space. Lemma 2.1 implies that $n_{c}^{g}$ is also independent of $g$ if any two $c$-good metrics can be connected by a smooth 1-parameter family of $c$-good metrics.

The numbers $n_{c}:=n_{c}^{g}$ associated to such almost canonical classes are called the mod 2-Seiberg-Witten invariants, and the classes $c$ with $n_{c} \neq 0$ are then called mod 2-Seiberg-Witten classes of index 0. By definition they are differentiable invariants, in the following sense: If $f: X^{\prime} \longrightarrow X$ is an orientation-preserving diffeomorphism, and for an almost canonical class $c$ of $X$ the Seiberg-Witten invariant $n_{c}$ is well defined, then $f^{*}(c)$ has the same property, and $n_{f^{*}(c)}=n_{c}$.

Remark 2.2 Let $c$ be an almost canonical class of $X$.

1. If $c^{2} \geq 0$ and $c_{\mathrm{DR}} \neq 0$, then any Riemannian metric on $X$ is c-good.

2. If $b_{2}^{+} \geq 2$, then any two c-good metrics can be connected by a smooth path of c-good metrics.

Therefore, if one of the two conditions above is satisfied, then the mod 2-Seiberg-Witten invariant $n_{c}$ is well-defined.

In the case $b_{2}^{+}=1$, invariants can still be defined, but the dependence of $n_{c}^{g}$ on the metric $g$ must be taken into account: In the real vector space $H_{\mathrm{DR}}^{2}(X)$, consider the positive cone

$$
\mathcal{K}=\left\{u \in H_{\mathrm{DR}}^{2}(X) \mid u^{2}>0\right\} .
$$

Fix a non-vanishing cohomology class $k \in H_{\mathrm{DR}}^{2}(X)$ with $k^{2} \geq 0$. The cone $\mathcal{K}$ splits as the disjoint union of its connected components $\mathcal{K}_{ \pm}$, where

$$
\mathcal{K}_{ \pm}:=\{u \in \mathcal{K} \mid \pm u \cdot k>0\} .
$$

If $c$ is an almost canonical class, let $c^{\perp}$ be the hyperplane

$$
c^{\perp}:=\left\{u \in H_{\mathrm{DR}}^{2}(X) \mid c \cdot u=0\right\}
$$


If $c^{\perp}$ meets $\mathcal{K}_{+}$, then the intersection $c^{\perp} \cap \mathcal{K}_{+}$is called the wall of type $c$, and the two components of $\mathcal{K}_{+} \backslash c^{\perp}$ are called chambers of type $c$. For every Riemannian metric $g$ on $X$, let $\omega_{g}$ be a generator of the real line of $g$ harmonic selfdual 2-forms, such that $\left[\omega_{g}\right] \in \mathcal{K}_{+}$. Then the ray $\mathbb{R}_{>0}\left[\omega_{g}\right] \subset \mathcal{K}_{+}$ depends smoothly on the metric $g$. The property of a metric to be $c$-good has the following simple geometric interpretation:

\section{Remark 2.3}

Suppose $b_{2}^{+}(X)=1$. Then:

1. The metric $g$ is c-good iff the ray $\mathbb{R}_{>0}\left[\omega_{g}\right]$ does not lie in the wall $c^{\perp} \cap \mathcal{K}_{+}$.

2. If $g_{0}$, and $g_{1}$ are c-good metrics, then $n_{c}^{g_{0}}=n_{c}^{g_{1}}$ iff the two rays $\mathbb{R}_{>0}\left[\omega_{g_{i}}\right]$ belong to the same chamber of type $c$.

The first assertion follows immediately from the definition. The second needs a careful analysis of a 1-parameter family of 0-dimensional smooth moduli spaces $\mathcal{W}_{X}^{g_{t}}(c)$ around the value of the parameter $t$ for which the ray $\mathbb{R}_{>0}\left[\omega_{g_{t}}\right]$ crosses the wall $c^{\perp} \cap \mathcal{K}_{+}$(see $[18],[15]$ ).

\section{Monopoles on Kähler surfaces}

Let $(X, J, g)$ be an almost complex 4-manifold endowed with a Hermitian metric $g$. The almost complex structure $J$ defines a reduction of the structure group of the tangent bundle $T_{X}$ of $X$ from $\mathrm{SO}(4)$ to $\mathrm{U}(2)$. In particular, we get a canonical $\operatorname{Spin}^{c}(4)$-structure on $X$ via the canonical lifting $l: \mathrm{U}(2) \longrightarrow \operatorname{Spin}^{c}(4)[11]$. Let $\omega_{g}$ be the Kähler form of $g$.

Lemma 3.1 [11] The canonical Spin $^{c}$-structure of an almost complex Hermitian 4-manifold has the following properties:

1. There are canonical identifications $\Sigma^{+}=\Lambda^{00} \oplus \Lambda^{02}, \Sigma^{-}=\Lambda^{01}$.

2. Via these identifications, the map $\Gamma: \Lambda_{+}^{2} \otimes \mathbb{C} \longrightarrow \operatorname{End}_{0}\left(\Sigma^{+}\right)$is given by:

$$
\Lambda^{20} \oplus \Lambda^{02} \oplus \Lambda^{00} \omega_{g} \ni\left(\lambda^{20}, \operatorname{lambda} a^{02}, f \omega_{g}\right) \stackrel{\Gamma}{\longrightarrow}\left[\begin{array}{cc}
-i f & -*\left(\lambda^{20} \wedge \cdot\right) \\
\lambda^{02} \wedge & \text { if }
\end{array}\right] \in \operatorname{End}_{0}\left(\Lambda^{00} \oplus \Lambda^{02}\right) .
$$

Suppose now that $(X, J, g)$ is a Kähler surface. This means that $J$ is integrable, and $\omega_{g}$ is closed (or equivalently, $J$ is Levi-Civita parallel). In particular the holonomy group of the Levi-Civita connection also reduces to $\mathrm{U}(2)$, and the splittings $\Lambda^{p} \otimes \mathbb{C}=\bigoplus_{i+j=p} \Lambda^{i j}$ are Levi-Civita parallel. We get a 
$\mathrm{U}(2)$-connection in the holomorphic tangent bundle $\mathcal{T}_{X}=T_{X}^{10} \simeq \Lambda^{01}$, which coincides with the Chern connection of this bundle, i.e. with the unique connection compatible with the holomorphic structure and the Hermitian metric. The induced connection $c_{0}$ in the line bundle $K_{X}^{\vee}=\operatorname{det}\left(\mathcal{T}_{X}\right) \simeq \Lambda^{02}$ also coincides with the Chern connection of this Hermitian holomorphic line bundle.

Every other $\operatorname{Spin}^{c}(4)$ structure $\hat{P}_{c} \longrightarrow P$ on $(X, g)$ has as spinor bundle

$$
\Sigma_{c}=\Sigma \otimes M
$$

where $M$ is a differentiable $S^{1}$-bundle with $2 c_{1}(M)+c_{1}\left(K_{X}^{\vee}\right)=c$. (For a simply connected manifold $X, M$ is well defined up to isomorphy by this condition.) $S^{1}$-connections in $\operatorname{det}\left(\Sigma_{c}^{ \pm}\right)=K_{X}^{\vee} \otimes M^{\otimes 2}$ correspond to $S^{1}$-connections in $M$. Given $b \in \mathcal{A}(M)$, the curvature of the corresponding connection $a \in \mathcal{A}\left(K_{X}^{\vee} \otimes M^{\otimes 2}\right)$ is $F_{a}=F_{c_{0}}+2 F_{b}$.

A half-spinor $\Psi \in A^{0}\left(\Sigma^{+} \otimes M\right)$ can be written as

$$
\Psi=\varphi+\alpha, \quad \varphi \in A^{0}(M), \quad \alpha \in A^{02}(M) .
$$

We put $J(M):=c_{1}\left(\Sigma^{+} \otimes M\right) \cup\left[\omega_{g}\right]$.

Proposition 3.2 Let $(X, g)$ be a Kähler surface with Chern connection $c_{0}$ in $K_{X}^{\vee}, M$ a differentiable $S^{1}$-bundle with $J(M)<0$. A pair $(b, \varphi+\alpha) \in$ $\mathcal{A}(M) \times\left(A^{0}(M) \oplus A^{02}(M)\right)$ solves the monopole equations iff:

$$
\begin{aligned}
& F_{b}^{20}=F_{b}^{02}=0 \\
& \alpha=0, \quad \bar{\partial}_{b}(\varphi)=0 \\
& i \Lambda F_{b}+\frac{1}{2} \varphi \bar{\varphi}+\frac{s}{2}=0 .
\end{aligned}
$$

Proof: The pair $(b, \varphi+\alpha)$ solves the equations $(S W)$ iff the corresponding pair $(a, \varphi+\alpha)$ satisfies

$$
\begin{array}{ll}
F_{a}^{20} & =-\varphi \otimes \bar{\alpha} \\
F_{a}^{02} & =\alpha \otimes \bar{\varphi} \\
\bar{\partial}_{b}(\varphi) & =i \Lambda \partial_{b}(\alpha) \\
i \Lambda F_{a} & =-(\varphi \bar{\varphi}-*(\alpha \wedge \bar{\alpha})) .
\end{array}
$$

By Corollary 1.7 it follows that $(b, \varphi+\alpha)$ solves $(S W)$ iff $(b, \varphi-\alpha)$ does (Witten's trick). Therefore $\varphi \otimes \bar{\alpha}=\alpha \otimes \bar{\varphi}=0$, hence $F_{a}^{20}=F_{a}^{02}=0$, and $\varphi$ 
or $\alpha$ must vanish. Integrating the equation $i \Lambda F_{a}=-(\varphi \bar{\varphi}-*(\alpha \wedge \bar{\alpha}))$ over $X$, we find:

$$
J(M)=\left(2 c_{1}(M)-c_{1}\left(K_{X}\right)\right) \cup\left[\omega_{g}\right]=\int_{X} \frac{i}{2 \pi} F_{a} \wedge \omega_{g}=\frac{1}{8 \pi} \int_{X}\left(-|\varphi|^{2}+|\alpha|^{2}\right),
$$

hence $\alpha=0$ if $J(M)<0$.

The above proposition must be interpreted as follows: If $J(M)<0$, then the solutions of the monopole equations $(S W)$ are the pairs $(b, \varphi) \in \mathcal{A}(M) \times$ $A^{0}(M)$, such that $b$ is the Chern connection of a holomorphic structure in $M, \varphi$ is a holomorphic section, and the mean curvature $i \Lambda F_{b}$ of $b$ satisfies the generalized vortex equation [16], [3], [4], [9]

$$
i \Lambda F_{b}+\frac{1}{2} \varphi \bar{\varphi}+\frac{s}{2}=0 .
$$

Moreover, every infinitesimal deformation of a solution of the form $(b, \varphi)$, $\varphi \neq 0$ of the monopole equation still vanishes in the $\alpha$-direction. Therefore $\mathcal{W}_{X}^{g}(c)$ can be identified (as real analytic space) with the moduli space of pairs $(b, \varphi)$ satisfying the above conditions, modulo the gauge group $\mathcal{C}^{\infty}\left(X, S^{1}\right)$ of unitary automorphisms of $M$. Under the assumption $J(M)<0$, the action of the gauge group is free on the space of solutions, because any solution $(b, \varphi)$ has a non-vanishing section $\varphi$.

Alternatively, let $\mathcal{M}$ be a holomorphic line bundle with differentiable support $M$, and $\varphi$ a holomorphic section of $\mathcal{M}$. For a Hermitian metric $h$ in $\mathcal{M}$, we denote by $F_{h}$ the curvature of the associated Chern connection, and we consider the following equation for $h$ :

$$
i \Lambda F_{h}+\frac{1}{2} \varphi \bar{\varphi}^{h}+\frac{s}{2}=0
$$

Standard arguments (see for instance [16], [9]) show that the problem of classifying the solutions $(b, \varphi)$ of $(*)$ modulo unitary automorphisms of $M$ is equivalent to the problem of classifying those pairs $(\mathcal{M}, \varphi)$ modulo holomorphic isomorphisms, for which the equation $\left(V_{s}^{\prime}\right)$ has a solution.

Proposition 3.3 Let $(X, g)$ be a compact Kähler surface, $(\mathcal{M}, \varphi)$ a holomorphic line bundle with a non-vanishing holomorphic section $\varphi \in H^{0}(X, \mathcal{M})$. $\mathcal{M}$ admits a metric $h$ satisfying the equation $\left(V_{s}^{\prime}\right)$ iff

$$
c_{1}(\mathcal{M}) \cup\left[\omega_{g}\right]<\frac{1}{2} c_{1}\left(K_{X}\right) \cup\left[\omega_{g}\right] .
$$


Proof: (cf. [3]) Fix a background metric $h_{0}$; any other metric $h$ has the form $h=e^{2 u} h_{0}$, with $u \in A^{0}$ a smooth function. The vortex equation $\left(V_{s}^{\prime}\right)$ translates into

$$
\Delta u+\frac{1}{2}|\varphi|_{h_{0}}^{2} e^{2 u}+\left(i \Lambda F_{h_{0}}+\frac{s}{2}\right)=0 .
$$

Set $q:=\int_{X}\left(i \Lambda F_{h_{0}}+\frac{s}{2}\right)=2 \pi\left(c_{1}(\mathcal{M})-\frac{1}{2} c_{1}\left(K_{X}\right) \cup\left[\omega_{g}\right]\right.$, and choose $v \in A^{0}$ with

$$
-\Delta v=\left(i \Lambda F_{h_{0}}+\frac{s}{2}\right)-q .
$$

Define $w:=2(u-v)$. Then (1) is equivalent to the following equation in $w$ :

$$
\Delta w+\left(|\varphi|_{h_{0}}^{2} e^{2 v}\right) e^{w}+2 q=0 .
$$

Integrating over $X$, we see that if (2) has solutions, then $q$ must be negative. On the other hand, by a well known result of Kazdan and Warner [3], (2) has a unique solution if $q<0$.

Theorem 3.4 [18], [16] Let $(X, g)$ be a simply connected Kähler surface, $c \in H^{2}(X, \mathbb{Z})$ with $c \equiv c_{1}\left(K_{X}\right) \bmod 2$, and $\pm c \cup\left[\omega_{g}\right]<0$.

1. If $c \notin \mathrm{NS}(X)$, then $\mathcal{W}_{X}^{g}(c)=\emptyset$.

2. Suppose $c \in \mathrm{NS}(X)$. Then there is a natural real analytic isomorphism $\mathcal{W}_{X}^{g}(c) \simeq \mathbb{P}\left(H^{0}(X, \mathcal{M})\right.$ ), where $\mathcal{M}$ is the (unique, up to isomorphy) holomorphic line bundle with $c_{1}\left(K_{X}^{\vee} \otimes \mathcal{M}^{\otimes 2}\right)= \pm c$.

3. $\mathcal{W}_{X}^{g}(c)$ is always smooth. Let $D$ be the divisor of a nontrivial section in $\mathcal{M}$. Then $\mathcal{W}_{X}^{g}(c)$ has the expected dimension iff $h^{1}\left(\left.\mathcal{O}_{X}(D)\right|_{D}\right)=0$.

\section{Rationality of complex surfaces}

A compact complex surface is rational iff its field of meromorphic functions is isomorphic to $\mathbb{C}(u, v)$. Such a surface is always simply connected and has $b_{2}^{+}=1[2]$. The following result has been has been announced by $\mathrm{R}$. Friedman and Z. Qin [8]. Whereas their proof uses Donaldson theory and vector bundles techniques, our proof uses the new Seiberg-Witten invariants, and our interpretation of these invariants in terms of linear systems.

Theorem 4.1 [17] A complex surface $X$ which is diffeomorphic to a rational surface is rational. 
Proof: The proof consists of the following three steps:

1.Any rational surface $X_{0}$ admits a Hitchin metric [12], i.e. a Kähler metric $g_{0}$ with positive total scalar curvature. This condition can be written as $c_{1}\left(K_{X_{0}}\right) \cup\left[\omega_{g_{0}}\right]<0$.

Let $c$ be any integral lift of $w_{2}\left(X_{0}\right)$, such that $g_{0}$ is $c$-good, i.e. such that the moduli space $\mathcal{W}_{X_{0}}^{g_{0}}(c)$ contains no reducible solutions. Since $p_{g}\left(X_{0}\right)=0$, $c$ has always type $(1,1)$, and $g_{0}$ is $c$-good iff $c \cup\left[\omega_{g_{0}}\right] \neq 0$.

We assert that $\mathcal{W}_{X_{0}}^{g_{0}}(c)$ is then empty, and in particular, all Seiberg-Witten invariants $n_{c}^{g_{0}}$ computed with respect to this metric vanish.

Indeed, let $\mathcal{M}$ be the holomorphic line bundle defined in Theorem 3.4. If the moduli space $\mathbb{P}\left(H^{0}\left(X_{0}, \mathcal{M}\right)\right)$ was not empty, then

$$
c_{1}(\mathcal{M}) \cup\left[\omega_{g_{0}}\right] \geq 0 .
$$

But we have

$$
0> \pm c \cup\left[\omega_{g_{0}}\right]=\left(2 c_{1}(\mathcal{M})-c_{1}\left(K_{X_{0}}\right)\right) \cup\left[\omega_{g_{0}}\right],
$$

hence, by (1)

$$
0 \leq 2 c_{1}(\mathcal{M}) \cup\left[\omega_{g_{0}}\right]<c_{1}\left(K_{X_{0}}\right) \cup\left[\omega_{g_{0}}\right],
$$

which contradicts the assumption on the total scalar curvature of $g_{0}$.

2. Let now $X$ be a simply connected projective surface with $\operatorname{kod}(X)>0$. We may suppose that $X$ is the blow up in $k$ distinct points of its minimal model $X_{\min }$. Denote by $\sigma: X \longrightarrow X_{\min }$ the contraction to the minimal model, and by $E=\sum_{i=1}^{k} E_{i}$ the exceptional divisor. Fix an ample divisor $H_{\text {min }}$ on $X_{\min }$, set $H_{n}:=\sigma^{*}\left(n H_{\min }\right)-E$, and for $n \gg 0$ choose a Kähler metric $g_{n}$ on $X$ with $\left[\omega_{g_{n}}\right]=c_{1}\left(H_{n}\right)$. Given $I \subset\{1, \ldots, k\}$, define

$$
\begin{aligned}
& E_{I}:=\sum_{i \in I} E_{i} \\
& c_{I}:=2 c_{1}\left(E_{I}\right)-c_{1}\left(K_{X}\right) \\
& \bar{I}:=\{1, \ldots, k\} \backslash I .
\end{aligned}
$$

Since $c_{I}$ is an almost canonical class, the expected dimension of the corresponding Seiberg-Witten moduli space is 0 . For $n \gg 0$ we get $c_{I} \cup\left[\omega_{g_{n}}\right]<0$, and Theorem 3.4 gives

$$
\mathcal{W}_{X}^{g_{n}}\left(c_{I}\right) \simeq\left\{E_{I}\right\}
$$


Therefore $\mathcal{W}_{X}^{g_{n}}\left(c_{I}\right)$ consists of a single smooth point, and

$$
n_{c_{I}}^{g_{n}}=1 \bmod 2 .
$$

3. Suppose now that there is an orientation-preserving diffeomorphism $f: X \longrightarrow X_{0}$, where $X$ is projective surface with $\operatorname{kod} X \geq 0$. Since $X$ must have $p_{g}(X)=0$, and $\pi_{1}(X)=\{1\}$, it follows that, in fact, $\operatorname{kod} X>0$. Let $g=f^{*}\left(g_{0}\right)$ denote the pull-back of a Hitchin metric to $X$; clearly

$$
n_{c_{I}}^{g}=0
$$

for all $I \subset\{1, \ldots, k\}$ such that $g$ is $c_{I^{-} \text {good. }}$

We will now derive a contradiction in the following way: Using the Enriques-Kodaira classification of surfaces, it easy to see that the de Rham cohomology class $k_{\min }:=\sigma^{*}\left(c_{1, \mathrm{DR}}\left(K_{\min }\right)\right)$ is non-trivial and satisfies the condition $k_{\min }^{2} \geq 0$. Therefore we can consider the upper positive cone

$$
\mathcal{K}_{+}:=\left\{u \in H_{\mathrm{DR}}^{2}(X) \mid u^{2}>0, u \cdot k_{\min }>0\right\} .
$$

Clearly $\left[\omega_{g_{n}}\right]$ belongs to $\mathcal{K}_{+}$. We choose a harmonic $g$-selfdual form $\omega_{g}$, with $\left[\omega_{g}\right] \in \mathcal{K}_{+}$.

Claim: The rays $\mathbb{R}_{>0}\left[\omega_{g}\right]$ and $\mathbb{R}_{>0}\left[\omega_{g_{n}}\right]$ belong either to the same chamber of type $c_{I}$ or to the same chamber of type $c_{\bar{I}}$.

Proof: If not, then, since $c_{I} \cup\left[\omega_{g_{n}}\right]<0$, we get $\left[\omega_{g}\right] \cdot c_{I} \geq 0$ and $\left[\omega_{g}\right] \cdot c_{\bar{I}} \geq 0$. Write

$$
\left[\omega_{g}\right]=\sum_{i=1}^{k} \lambda_{i} E_{i}+\sigma^{*}[\omega]
$$

with $[\omega] \in H_{\mathrm{DR}}^{2}\left(X_{\min }\right)$. Then

$$
\begin{aligned}
& -\sum_{i \in I} \lambda_{i}+\sum_{j \in \bar{I}} \lambda_{j}-[\omega] \cdot\left[K_{\min }\right] \geq 0 \\
& -\sum_{j \in \bar{I}} \lambda_{j}+\sum_{i \in I} \lambda_{i}-[\omega] \cdot\left[K_{\min }\right] \geq 0 .
\end{aligned}
$$

Adding these inequalities we find $[\omega] \cdot\left[K_{\min }\right] \leq 0$. But $[\omega] \cdot\left[K_{\min }\right]=\left[\omega_{g}\right] \cdot k_{\min }>$ 0 , because $\left[\omega_{g}\right] \in \mathcal{K}_{+}$. This contradiction proves the claim.

It follows that either $g$ and $g_{n}$ are both $c_{I^{-}}$good and $n_{c_{I}}^{g}=n_{c_{I}}^{g_{n}}$, or $g$ and $g_{n}$ are both $c_{\bar{I}}$-good and $n_{c_{\bar{I}}}^{g}=n_{c_{\bar{I}}}^{g_{n}}$. This gives now a contradiction with (2) and (3).

Together with the results of Friedman and Morgan [7], we have: 
Theorem 4.2 (The Van de Ven conjecture [19]) The Kodaira dimension of complex surfaces is a $\mathcal{C}^{\infty}$-invariant.

Remark: It is possible to couple the Seiberg-Witten equations to connections in unitary bundles. The solutions of these coupled Seiberg-Witten equations over Kähler surfaces again have a purely complex-geometric interpretation [16]: The moduli space of solutions can be identified-via generalized vortex equations - with moduli spaces of stable pairs [13], [4]. This construction could lead to new invariants which might be nontrivial for Kähler surfaces wit $p_{g}=0$.

\section{Bibliography}

1. Atiyah M., Hitchin N. J., Singer I. M.: Selfduality in four-dimensional Riemannian geometry, Proc. R. Lond. A. 362, 425-461 (1978)

2. Barth, W., Peters, C., Van de Ven, A.: Compact complex surfaces, Springer Verlag (1984)

3. Bradlow, S. B.: Vortices in holomorphic line bundles over closed Kähler manifolds, Comm. Math. Phys. 135, 1-17 (1990)

4. Bradlow, S. B.: Special metrics and stability for holomorphic bundles with global sections, J. Diff. Geom. 33, 169-214 (1991)

5. Donaldson, S.; Kronheimer, P. B.: The Geometry of four-manifolds, Oxford Science Publications (1990)

6. Freed, D. S.; Uhlenbeck, K. K.: Instantons and Four-Manifolds, Springer Verlag (1984)

7. Friedman, R., Morgan, J.W.: Smooth 4-manifolds and Complex Surfaces, Springer Verlag 3. Folge, Band 27 (1994)

8. Friedman, R., Qin, Z.: On complex surfaces diffeomorphic to rational surfaces, Preprint (1994)

9. Garcia-Prada, O.: Dimensional reduction of stable bundles, vortices and stable pairs, Int. J. of Math. Vol. 5, No 1, 1-52 (1994)

10. Hirzebruch, F., Hopf H.: Felder von Flächenelementen in 4-dimensionalen 4-Mannigfaltigkeiten, Math. Ann. 136, (1958)

11. Hitchin, N.: Harmonic spinors, Adv. in Math. 14, 1-55 (1974)

12. Hitchin, N.: On the curvature of rational surfaces, Proc. of Symp. in Pure Math., Stanford, Vol. 27 (1975) 
13. Huybrechts, D.; Lehn, M.: Stable pairs on curves and surfaces, J. Alg. Geometry, (1995)

14. Kobayashi, S.: Differential geometry of complex vector bundles, Princeton University Press, (1987)

15. Kronheimer, P., Mrowka, T.: The genus of embedded surfaces in the projective plane, Preprint (1994)

16. Okonek, Ch.; Teleman A.: The Coupled Seiberg-Witten Equations, Vortices, and Moduli Spaces of Stable Pairs, Preprint, January, 13-th 1995

17. Okonek, Ch.; Teleman A.: Seiberg-Witten invariants and the Van de Ven conjecture, Preprint, February, 8-th 1995

18. Witten, E.: Monopoles and four-manifolds, Mathematical Research Letters 1, 769-796 (1994)

19. Van de Ven, A,: On the differentiable structure of certain algebraic surfaces, Sém. Bourbaki nº 667, Juin (1986)

Authors addresses:

Mathematisches Institut, Universität Zürich,

Winterthurerstrasse 190, CH-8057 Zürich

e-mail:okonek@math.unizh.ch

teleman@math.unizh.ch 